\title{
Self-Regulation and Mechanisms of Action in Psychotherapy: A Theory-Based Translational Perspective
}

\author{
Timothy J. Strauman,' Elena L. Goetz,' \\ Allison M. Detloff,' Katherine E. MacDuffie,' \\ Luisa Zaunmüller, ${ }^{2}$ and Wolfgang Lutz ${ }^{2}$ \\ 'Duke University \\ ${ }^{2}$ University of Trier
}

\begin{abstract}
Psychotherapy is a complex, multilayered process with the potential to bring about changes at multiple levels of functioning, from the neurobiology of the brain to the individual's role in the social world. Although studies of the mechanisms by which psychotherapy leads to change continue to appear, there remains much to be learned about how psychological interventions work. To guide explorations of how and for whom particular treatment approaches lead to change, researchers can rely on theory to identify potential loci for change and on translational research methods to integrate basic behavioral science and neuroscience with clinical science. In this article, we describe research linking individual differences in the self-regulation of personal goal pursuit with the etiology and treatment of mood disorders. The research draws upon regulatory focus theory as a model of self-regulation and on microintervention designs-controlled laboratory investigations of a specific therapeutic technique-to generate and test hypotheses about how psychological interventions can help to reverse maladaptive selfregulatory processes.
\end{abstract}

Words are the physicians of a mind diseased.-Aeschylus

The "talking cure" has been available as a formal treatment modality for psychological disorders for more than a century, and there is substantial evidence that psychotherapy is effective in reducing distress and increasing adaptive functioning (e.g., Lambert, 2011). Nonetheless, as codified by Gordon Paul (1967, p. 211), there remains much to be learned about "what treatment, by whom, is most effective for this individual with that specific problem, under which set of circumstances." The evidence-based treatment movement has brought muchneeded attention to the impressive overall efficacy of psychotherapy, but has not yet answered the critical questions articulated by Paul more than 40 years ago (Kazdin, 2011). In particular, relatively little is known about how to tailor psychological interventions to the characteristics and needs of specific individuals (Norcross \& Wampold, 2011).

As the study of psychotherapeutic mechanisms of action and treatment matching proceeds, it is important to recognize that behavioral science and its cognate discipline, neuroscience, each have enormous relevance for understanding psychotherapy specifically and behavior change more generally (Cacioppo, Berntson, Sheridan, \& McClintock, 2000). While there has been progress toward a more individualized understanding of vulnerability to psychopathology (Belsky \& Pluess, 2009), the application of individual differences theory and research to psychotherapy remains in its infancy (Beutler, 2011). Furthermore, without the guidance of theory and data, it will likely be impossible to establish principles by which to maximize psychotherapy efficacy and identify relevant mechanisms of action that apply across individuals. Fortunately, both behavioral science and neuroscience provide conceptual models that are translatable into psychotherapeutic interventions (Mischel, 2004). Similarly, many of the factors implicated in treatment efficacy have been studied extensively by basic scientists from each discipline (Strauman, Klenk, \& Eddington, in press). Each science can provide theory-based,

This research was supported by National Institute of Mental Health (NIMH) Grant R24 MH67447 and National Institute on Drug Abuse (NIDA) Grant P30 DA023026, by R0I DA03 1579, which is supported by the NIH Common Fund and managed by the OD/Office of Strategic Coordination, and by funding from the Humboldt Foundation.

Correspondence concerning this article should be addressed to Timothy J. Strauman, Department of Psychology and Neuroscience, Duke University, 417 Chapel Drive, Box 90086, Durham, NC 27708.

Email: tjstraum@duke.edu. 
empirically substantiated targets for change in therapy as well as innovative methods for assessing change. Such models, in turn, may provide frameworks for treatment matching, which is ideally a systematic a priori process by which a treatment is selected as most likely to provide maximum benefit for a particular individual (Beutler, 2011).

In this article, we describe how theory-based microintervention research-controlled investigations of a specific psychotherapeutic technique guided by a model of change processes - provides a unique opportunity to test translational hypotheses about how psychotherapy works and for whom it works. This approach combines methods and hypotheses derived from basic science with psychological theories that guide their application. Our own research draws upon regulatory focus theory (RFT; Higgins, 1998) as a model of self-regulation that is highly applicable to research on psychotherapy. We report on recent investigations exploring the role of individual differences in the self-regulation of personal goal pursuit within the treatment of mood disorders. Although to date there has been little overlap between studies of selfregulation and microintervention research methods, we hope to illustrate how combining them can be especially useful for advancing our understanding of what works for whom within the domain of psychological interventions. And in doing so, we hope to demonstrate that psychological constructs and principles need to be at the core of research investigating how and for whom psychotherapy works (Miller, 2010).

\section{The Role of Theory in Studying How Psychotherapy Works}

Translational research, defined as the transfer of basic knowledge in behavioral science and neuroscience into the development of new methods for diagnosis, treatment, and prevention of mental disorders (Sung et al., 2003), has become increasingly important in both research funding and mental health policy. At the National Institutes of Health, for example, there has been a radical "re-engineering" of clinical research support to take better advantage of newly emerging knowledge from basic science domains (NIH, 2007). However, in contrast to the field of medicine, where translational research is guided primarily by discovery (Woolf, 2008), both behavioral science and neuroscience are translated into clinical interventions most efficiently when guided by theory (National Institute of Mental Health, 2000). Neuroscience and behavioral science both are primarily Galilean sciences, meaning that they are organized around hypothetical constructs that underlie and find expression in observed phenomena and that they ultimately rely on theories as statements of lawful associations between constructs (Popper, 1979). Thus, the translation of neuroscience and behavioral science knowledge into models for psychotherapy research relies on theories that stipulate how constructs are related (Forsyth \& Strong, 1986). The list of psychological constructs relevant to psychotherapy process is long and impressive-familiar examples include attachment (Connors, 2011), emotion regulation (Greenberg, 2008), conditioning (Zinbarg \& Griffith, 2008), and insight (Caspar, 1997), to name a few.

Surprisingly, there has been considerable debate among psychotherapists regarding whether psychological interventions need to be centered upon basic science. For example, efforts to apply neuroscience and neuroimaging findings to understanding psychotherapy mechanisms of action have been viewed as controversial and even counterproductive, and concerns have been raised about whether such knowledge can be translated into psychotherapy practice (for a thoughtful discussion, see Caspar, 2003). It is our contention (Strauman et al., in press), along with others (e.g., Brenner, Roder, \& Tschacher, 2006; Etkin, Pittenger, Polan, \& Kandel, 2005; Grawe, 2007; Linden, 2006; Roffman, Marci, Glick, Dougherty, \& Rauch, 2005), that both behavioral science and neuroscience models and findings have a central place in the study of psychotherapy mechanisms of action. The key is to link relevant theory with carefully selected experimental tasks and populations of interest, in order to maximize the translational potential from basic to clinical science and back again (Carrig, Kolden, \& Strauman, 2009). The microintervention research strategies we will describe, as well as the self-regulation model of depression and its application in psychotherapy research to date, are both intended to facilitate a theory-based translational approach that will contribute to our understanding of how and for whom psychotherapy works.

\section{Microintervention Studies in Translational Psychotherapy Research}

Psychotherapy research differentiates between specific and common factors that contribute to the therapeutic outcome. Common (also called universal or transtheoretical) factors are those which are postulated to operate in all psychotherapies and are not limited to a particular theory, model, or technique. Such factors as the quality of the working alliance between therapist and patient, the development of a common understanding of the problems facing the patient, and a commitment to work together to improve the patient's functioning all represent well-documented processes associated with successful outcomes in many types of psychotherapy (e.g. Castonguay, 1993). There is a growing number of studies examining how such factors influence brain activation while simultaneously influencing self-awareness and engagement in therapy (Beitman, Viamontes, Soth, \& Nittler, 2006). In addition to these universal processes, there also are techniques and processes associated with particular theories or models of psychotherapy which are hypothesized to contribute significantly to treatment efficacy (e.g., Clark et al., 2006).

To investigate change mechanisms in psychotherapy, it may not be optimal to use traditional treatment research designs such as randomized clinical trials with homogeneous patient 
groups (efficacy studies) or naturalistic designs in community settings (effectiveness studies) (Lutz, Stulz, \& Köck, 2009). Treatment-focused designs assess the impact of a particular psychotherapy as a whole (i.e., as a "package"), but such designs often cannot discriminate the impact of specific putative mechanisms of change. An alternative strategy for investigating specific factors or techniques is to study microinterventions that target smaller units of cause and effect in psychotherapeutic settings. Microinterventions are discrete, time-limited applications of a single psychotherapeutic technique. Microinterventions can be studied within controlled experimental designs, enabling the researcher to draw conclusions about whether and how specific techniques may lead to particular changes that are observed within a larger course of treatment (Zaunmüller, Lutz, \& Strauman, under review). To the extent that a microintervention study can balance theory, rigorous experimental design, and internal validity with applicability to the broader psychotherapy literature, the data can provide a useful conceptual and empirical bridge between efficacy and process studies (Lutz et al., 2005).

\section{An Example of a Microintervention Study: Cognitive Restructuring}

Current psychotherapies for mood and anxiety disorders (as well as other disorders) often incorporate techniques intended to identify, challenge, and alter dysfunctional beliefs and thought patterns (Dozois \& Beck, 2011). Although there is substantial evidence for the efficacy of cognitive-behavioral therapies as units of treatment (e.g., Hollon \& Ponniah, 2010), there remains a need for microanalytic studies examining how specific techniques may contribute to the overall efficacy of the treatment. Recently, DeRubeis and associates proposed a model for the neural mechanisms underlying cognitive therapy for depression (DeRubeis, Siegle, \& Hollon, 2008). Depressed individuals frequently manifest an increase in activation of the amygdala, a limbic region that is crucial for emotional processing and reactivity, as well as decreased activation in the prefrontal cortex (PFC), which plays an important role in cognitive control processes (Siegle, Thompson, Carter, Steinhauer, \& Thase, 2007). DeRubeis and colleagues proposed that cognitive therapy strengthens the inhibitory executive control processes associated with the PFC, which interrupt or dampen limbic activation associated with intense negative affect. According to this model, successful cognitive therapy should yield increased PFC activity and decreased amygdala activity. However, functional imaging studies of cognitive therapy have reported mixed findings (e.g., Goldapple et al., 2004), which might be due to differences in experimental designs, to the use of resting state paradigms or symptom provocation paradigms, or to a variety of other methodological differences (for a more detailed discussion see Linden, 2006).

There have been a number of behavioral studies investigating particular cognitive therapy techniques, such as cognitive restructuring, that can be used to generate hypotheses regarding the neural correlates of cognitive restructuring as a microintervention (Ochsner \& Gross, 2008). Cognitive restructuring of negative emotion-inducing stimuli activates the dorsal anterior cingulate cortex (ACC) and the PFC, both of which are associated with cognitive control processes and support the selection and application of restructuring strategies (DeRubeis, Siegle, \& Hollon, 2008). Activations in these cognitive control regions are hypothesized to produce a decrease of activity in emotional processing regions such as the amygdala or insula that is associated with a reduction in the intensity of negative affect.

Despite the excitement regarding the potential for adding a neural level of analysis to studies of psychotherapy mechanisms of action, the technical and logistical challenges of doing so can be daunting. Electroencephalography (EEG) is a particularly feasible methodology for investigating treatment effects, as the method is noninvasive, cost-effective, and provides excellent temporal resolution to detect neuronal changes. Several recent studies have measured event-related potentials (ERPs) while instructing participants to use brief restructuring techniques during exposure to negatively valenced stimuli. Hajcak and Nieuwenhuis (2006) investigated electrocortical changes associated with cognitive restructuring and the time course of associated neural modulations in an ERP study. Event-related brain potentials were recorded while participants were presented with negatively valenced affective pictures and instructed to either attend to or reappraise the unpleasant pictures. Late positive potential (LPP) amplitude in response to the negative pictures was significantly reduced for the reappraisal instruction condition. The LPP has been related in a number of previous studies to the emotional intensity or motivational significance of stimuli (e.g., Cuthbert, Schupp, Bradley, Birbaumer, \& Lang, 2000). Hajcak and Nieuwenhuis reported that reduced LPP within the cognitive restructuring condition was positively correlated with reductions in emotional intensity ratings, suggesting that reduced LPP represents an index of diminished negative affect.

Zaunmüller, Lutz, \& Strauman (under review) investigated the electrocortical correlates of cognitive restructuring, delivered as a 90-minute psychotherapeutic microintervention. The intervention consisted of instruction and demonstration of ways to reframe and reinterpret upsetting stimuli plus opportunities to practice the techniques with the assistance of the experimenter/therapist. Participants who reported either moderate or low levels of dysphoric symptoms were randomly assigned to a restructuring microintervention or to one of two comparison conditions. The effects of the experimental conditions were examined using recordings of event-related potentials as well as self-reported mood change. In the EEG paradigm, participants viewed unpleasant pictures, with the instruction to either reappraise the picture content or to simply attend to it - a paradigm that has been employed in a number of previous studies (e.g. Hajcak \& Nieuwenhuis, 2006; Phan et al., 2005). Following each trial, participants were asked to 
rate the intensity of their emotional response as well as their success of following the instruction to either "reframe" or to "attend."

Zaunmüller et al. (under review) found that the restructuring microintervention, compared to the two other conditions, had specific effects which were reliably detected on the ERP measures. The ERP findings were partly consistent with, but partly distinct from, observations from prior studies. For the LPP, they observed greater mean LPP amplitude for the "reframe" condition compared to the "attend" condition. LPP amplitudes for the "reframe" condition were generally larger in the left hemisphere and were largest at anterior and central electrode sites within the restructuring intervention group. These results contrast the findings of reduced LPP amplitudes in the reappraise condition found by Hajcak and Nieuwenhuis (2006).

The finding of increased frontal LPP amplitudes for the "reframe" compared to the "attend" condition, especially within the restructuring intervention group, could reflect an intervention-specific effect of strengthening inhibitory executive control processes which help to interrupt or dampen emotional arousal. This interpretation would be consistent with the DeRubeis et al. (2008) model of the neural mechanisms underlying cognitive therapy for depression. Positive potentials with maximal amplitudes in frontal brain regions have previously been associated with consciously controlled cognitive processing often described as executive function. Lewis, Lamm, Segalowitz, Stieben, \& Zelazo (2006) proposed a link between such processes and intentionally implemented emotion regulation strategies, suggesting that these potentials might be related to inhibitory top-down processes involved in cognitive emotion regulation mediated by the $\mathrm{PFC}$. This suggestion is consistent with current models concerning the neural substrates of emotion regulation (Kim \& Hamann, 2007; Taylor \& Liberzon, 2007), which propose that cognitive restructuring of negative affect is mediated by the PFC and related paralimbic structures such as the ACC which have inhibitory effects on emotional processing systems.

Zaunmüller et al. (under review) also found interventionspecific effects concerning the topographical distribution of the P300 (an ERP component consisting of a positive deflection that begins approximately 300 milliseconds after stimulus presentation). The P300 has been associated with emotional evaluation of a stimulus, as well as with "oddball" detection and with allocation of cognitive resources (Olofsson, Nordin, Sequeira, \& Polich, 2008). For the restructuring intervention group, Zaunmüller and colleagues found larger P300 amplitudes in the left hemisphere, in contrast to larger amplitudes in the right hemisphere for both comparison groups. Left hemisphere activation, particularly when originating from left frontal sites, has been associated with positive affect and approach-related motivational states, while right frontal activity is associated with negative affect and withdrawal-related motivational states (Davidson, Jackson, \& Kalin, 2000; Peterson, Gable, \& Harmon-Jones, 2008). With the instruction to "reframe," participants were asked to generate a less negative interpretation of the picture content which is likely linked to the generation of positive, approach-related emotionparticularly within the restructuring intervention, which provided training in such a strategy. In general, participants reported that they were able to reframe and reinterpret the content of the pictures on the basis of the single session of instruction and practice, and that they experienced less negative affect when they reinterpreted negatively valenced stimuli in this manner.

The Zaunmüller et al. (under review) study illustrates several important advantages of microintervention research. First, the study makes clear that with thoughtful design and careful attention to capturing the essential qualities of a psychotherapeutic technique, it is possible to use the behavioral or neuroscience laboratory to investigate the neural changes that accompany successful cognitive intervention strategies at a level of analysis that offers both rigorous experimental control and a reasonable analog to the actual work of therapist and patient. Second, microintervention research allows for more precise testing of theory-based predictions; in this instance, testing predictions regarding the acute impact of a simple but potentially powerful cognitive reframing technique. Within a traditional efficacy or effectiveness study, there may be no equivalently direct and rigorous way to determine the impact of the restructuring intervention. Third, the introduction of neuroscience techniques (here, ERP measures) brings an additional level of analysis into play that may help to illuminate how techniques work. The findings of this study suggest that the restructuring/reframing intervention influenced both topdown cognitive control and positive affectivity.

\section{Combining Theory and Microintervention Methods to Study Change Processes in Psychotherapy: Targeting Maladaptive Self-Regulation}

In the remainder of this article, we explore the combination of a theory-based translational perspective on how psychotherapy works - in this case, with RFT as an exemplar of theories describing self-regulation - with the use of microintervention designs to complement and extend conventional efficacy studies. We discuss how self-regulation is a ubiquitous and essential psychological process that represents a point of intersection for mind, brain, and the interpersonal world (Heatherton \& Wagner, 2011; Strauman, 2002). We then briefly review studies examining the impact of conventional treatments on self-regulatory function, and then introduce a therapy that attempts to translate the insights of RFT into a treatment for individuals whose depression is characterized by profound self-regulatory dysfunction. Finally, we describe two pilot studies testing predictions from RFT by means of microintervention designs. 


\section{Regulatory Focus Theory and Individual Differences in Vulnerability to Depression}

The history of psychology includes a succession of theories intended to account for the purposive, goal-driven nature of behavior and individual differences in goal pursuit (Bandura, 2006). Goals can be defined as internal representations of desired states, and approach and avoidance goals are among the most important classes of goals (Austin \& Vancouver, 1996; Elliot, 2008). Furthermore, different manifestations of psychopathology can be conceptualized as dysfunctions in the self-regulation of personal goal pursuit (Karoly, 1999). In this way, self-regulation constitutes a critical locus for the influence of motivation, cognition, emotion, and behavior on the psychological and physical health of the individual (Strauman, 2002).

Two related theories of self-regulation have been particularly influential in modeling vulnerability to psychopathology and treatment of psychological disorders: self-discrepancy theory (SDT; Higgins, 1987) and regulatory focus theory (Higgins, 1998). SDT is a model of self and affect which proposes that different relations between a person's representations of their actual behaviors and attributes (the actual self) and personally significant goals or standards (self-guides) have different motivational and emotional consequences. The actual self is a representation of the attributes that an individual believes he or she actually possesses. The ideal self is a representation of the attributes that an individual ideally would like to possess (their hopes, wishes, or aspirations). The ought self is a representation of the attributes that an individual believes it is his/her obligation or duty to possess. Patterns of relations between these self-state representations serve to indicate an individual's progress toward personal goals. It is important to note that personal attributes toward which individuals strive can be construed as ideals or as oughts. For example, for one person, the goal to be successful might represent an ideal or accomplishment, whereas for another the same goal might instead represent an obligation or responsibility.

According to SDT, discrepancies between the actual self and different self-guides lead to distinct negative emotional states. An actual-self/ideal-self discrepancy is hypothesized to lead to dejection-related emotions (such as sadness or disappointment) because such a perceived discrepancy signifies a failure to attain a hoped-for state. In contrast, an actual-self/ ought-self discrepancy is hypothesized to lead to agitationrelated emotions (such as anxiety, worry, or guilt) because such a perceived discrepancy signifies a failure to live up to one's responsibilities or obligations. Although the self-discrepancy literature contains some inconsistencies (e.g., Tangney, Niedenthal, Covert, \& Barlow, 1998), the theory's predictions have received considerable support.

RFT builds upon self-discrepancy theory to propose a more general psychological model of the cognitive and motivational processes that underlie the pursuit of desired end-states and the affective consequences of success versus failure. RFT distinguishes between self-regulation with respect to a promotion focus versus a prevention focus. Promotion represents a concern with advancement, growth, and accomplishment based on nurturance needs, strong ideals (strong aspirations and hopes for oneself), and situations that are framed in terms of the presence or absence of positive outcomes (gain/nongain). Promotion-focused self-regulation involves sensitivity to positive outcomes, use of an approach strategy for attaining desired end-states, and eagerness for advancement and gains (maximizing attainment, and ensuring against missed opportunities for attainment) as a means for pursuing personal goals - a motivational orientation that can be described as "making good things happen."

In contrast, prevention represents a concern with protection, safety, and responsibility based on security needs, strong oughts (a strong sense of one's duties and responsibilities), and situations framed in terms of the presence or absence of negative outcomes (loss/non-loss). Prevention-focused selfregulation involves a sensitivity to negative outcomes, use of an avoidance strategy for attaining desired end-states, and vigilance to ensure safety and non-loss (vigilance against making mistakes, and ensuring against committing the error of producing them) as a means for pursuing goals - a motivational orientation that can be described as "keeping bad things from happening."

RFT postulates that successful pursuit of a promotion goal (e.g., congruency or match with an ideal self-guide) represents the presence of a positive outcome, resulting in the experience of cheerfulness-related emotions such as happiness. In contrast, unsuccessful pursuit of a promotion goal (e.g., a discrepancy with an ideal-self guide) represents the absence of a positive outcome, resulting in the experience of dejectionrelated emotions and potentially dysphoric symptoms. The theory also postulates that successful pursuit of a prevention goal (e.g., congruency with an ought self-guide) represents the absence of a negative outcome, resulting in the experience of quiescence-related emotions such as relief or contentment, whereas unsuccessful pursuit of a prevention goal (e.g., a discrepancy with an ought self-guide) represents the presence of a negative outcome, resulting in the experience of agitationrelated emotions and potentially symptoms of anxiety. Higgins and Spiegel (2004) summarized the body of evidence in support of these predictions.

The regulatory focus perspective has been incorporated into recent models of mood and anxiety disorders. Strauman (2002) proposed a model of vulnerability to depression in which individuals experiencing chronic failure to attain promotion goals were viewed as especially vulnerable to mood disorder. Miller and Markman (2007) tested predictions of RFT in a sample of distressed undergraduates and observed that, as expected, promotion focus was negatively associated with symptoms of hopelessness depression among individuals who perceived themselves as failing to attain important personal goals. Because these theories of self-regulation offer specific hypoth- 
eses about how individuals with different self-regulatory concerns will respond to success and failure experiences, they serve as a useful framework for identifying therapeutic strategies that can be tailored to the individual. In this way, the theories are both overarching yet flexible in their applicability to individualized psychotherapy.

\section{Effects of Conventional Treatments for Depression on Self-Regulatory Cognition}

Existing treatments for depression with demonstrated efficacy appear to have equivalent rates of clinical effectiveness overall. However, simply knowing that treatments A, B, and C all lead to remission $60 \%$ of the time does not provide a reliable $a$ priori basis for determining which treatment would be most effective for a particular individual. One reason for this continuing dearth of knowledge is that the mechanisms by which the treatments lead to particular alterations in psychological processes implicated in the onset and maintenance of depression remain to be elucidated. Our research group has been examining similarities and differences across treatments in a number of studies over the past decade. For example, Strauman et al. (2001) reported two studies examining the effects of empirically supported treatments for depression on perceived failure in self-regulation (operationalized as self-discrepancy). In Study 1, patients received group cognitive-behavioral therapy (CBT); in Study 2, patients received either individual CBT or interpersonal psychotherapy (IPT) or pharmacotherapy. The treatments showed equivalent efficacy, but only CBT and IPT were associated with decreased self-discrepancy as well as decreased negative mood reactivity to idiographic ideal/ought goal priming. Perhaps more importantly, the investigators also observed that highly self-discrepant patients showed less improvement than other patients across all treatments, even after controlling for initial severity. The findings suggested that treatments may differ in their impact on selfregulatory cognition, and that certain individuals may require a treatment approach that offers a greater emphasis on selfregulation than conventional treatments.

\section{Self-System Therapy: A Theory-Based Therapy for Depression}

Our research group has developed and tested a self-regulationbased treatment for depression, self-system therapy (SST; Vieth, Strauman, Kolden, Woods, \& Klein, 2003). SST was designed to focus directly on self-regulatory dysfunction in depression and incorporates a number of specific strategies and interventions from efficacious treatments such as CBT, IPT, and behavioral activation therapy (Jacobson, Martell, \& Dimidjian, 2001). Drawing upon regulatory focus theory, we designed SST to be a modular treatment organized into three phases: an orientation to treatment (including initiation of goal-focused behavioral activation exercises as well as assessment of current and past significant relationships); an exploration of the individual's goals, standards, and self-regulatory style; and the development of a set of change/compensation sections targeting the individual's specific problems and vulnerabilities. In a randomized clinical trial comparing SST with CBT (Strauman et al., 2006), we observed that whereas the two treatments were equivalently efficacious overall, SST was more effective for patients who reported chronic difficulties attaining their ideal goals - consistent with our self-regulation model of depression. We also observed that SST was more effective than CBT at reducing patients' tendencies to recall negatively valenced life events when primed with personal goals - initial evidence that SST and CBT may differ in their characteristic mechanisms of action.

\section{Applying RFT to the Study of Psychotherapy via Microintervention Research}

Although the clinical trial data was consistent with the general assertion that depressed individuals manifesting substantial self-regulatory dysfunction might benefit more from a treatment specifically targeting that problem, clinical trials are inherently limited in their ability to elucidate how treatments work. As a follow-up to the clinical study, we have been engaged in translating aspects of RFT into novel techniques that clinicians could teach patients to use when experiencing different negative emotions. These techniques would be developed specifically to target hypothesized dysfunctions in the promotion and prevention systems in order to reduce dysphoric and anxious affect, respectively.

One such aspect of regulatory focus is the observation that the promotion and prevention systems have different motivational characteristics, which lead to a critical distinction with regard to psychopathology: dysphoric moods and clinical depression are characterized by inadequate engagement of the promotion system, whereas agitated moods and clinical anxiety are characterized by excessive engagement of the prevention system (Higgins, 2001). If the promotion system is underactive, the individual is likely to experience decreased approach motivation, loss of interest, anhedonia, and related symptoms. In contrast, if the prevention system is overactive, the individual is likely to experience agitation, hypervigilance, and worry. Following from these principles, we postulated that particular kinds of success experiences would be differentially helpful to individuals struggling with depressive versus anxious symptoms. Specifically, success in pursuing a promotion goal increases both positive mood and eagerness and thus would increase the individual's strength of engagement in promotion goal pursuit. Likewise, success in pursuing a prevention goal decreases both agitated mood and vigilance and thus decreases prevention engagement strength. As such, a therapist might want to have specific intervention techniques available that not only target promotion versus prevention but 
can increase engagement of the former system and decrease engagement of the latter.

In addition to encouraging patients to acknowledge their efforts and their successes in everyday life (as is done routinely in cognitive-behavioral therapy), what else might be done to bring about the needed changes in engagement strength for depression and anxiety? We proposed an additional approach that takes advantage of gains in knowledge about engagement strength and reframing experiences of adversity. Specifically, Higgins, Marguc, and Scholer (2012, p. 8) observed that the strategy of dealing with adversity by pushing back toward a goal that has been blocked ("opposing an interfering force") helps to re-engage the promotion system. On the other hand, the strategy of labeling the adversity as a nuisance rather than as a threat and simply coping with it without undue aggravation ("coping with a nuisance") helps to decrease prevention system engagement. We translated these observations from the self-regulation model into microinterventions to determine whether they had the predicted effects on acute distress.

Individuals experiencing chronic dysphoric and/or anxious symptoms participated in a one-session analog therapeutic intervention in which they were presented with either one or both of two scripts: a description of coping with distress by overcoming obstacles (intended to increase promotion engagement strength) or a description of viewing the distress as a nuisance (intended to decrease prevention engagement strength). After providing informed consent, participants completed the state version of the positive and negative affect schedule (PANAS) and a set of anagrams which provided a baseline for assessment of their characteristic strength of ideal and/or ought standards. Participants were then randomized to one of four intervention conditions: Increase Promotion Engagement (targeting overcoming obstacles and alleviating dysphoric symptoms), Decrease Prevention Engagement (targeting viewing distress as a nuisance and reducing anxious symptoms), Combined (both interventions), or an active control intervention targeting neither kind of symptoms. The script(s) was/were presented by the therapist/experimenter and discussed with the participant for approximately 20 minutes. The participant was encouraged to generate examples of current problematic situations and to apply the technique described in the script to those situations. After the intervention, participants then completed another PANAS and another set of anagram tasks to determine the acute impact of the interventions on mood as well as engagement strength.

A total of 66 participants completed the study. Table 1 summarizes the mood change (on the PANAS positive affect and negative affect scales) that was associated with the four intervention conditions. Analysis of variance with Time (preintervention, post-intervention) and PANAS Scale (positive and negative affect) as within-subject factors and Intervention Condition (Control, Increase Promotion, Decrease Prevention, Combined) as a between-subjects factor revealed a significant Time $\times$ PANAS Scale $\times$ Intervention Condition interaction, $F(2,150)=5.56, p<.01$. The interaction indicated that positive and negative affect changed differentially across the four intervention conditions. Planned post-hoc comparisons were conducted to test our specific hypotheses. We observed that for positive affect, the Increase Promotion Engagement and Combined conditions led to greater improvement than the other two conditions, $F(2,150)=4.87, p<.02$. Similarly, for negative affect, we observed that the Decrease Prevention Engagement and Combined conditions led to greater improvement (decreased negative affect) than the other two conditions, $F(2$, $150)=4.43, p<.02$.

Other recent studies examining the experiences of success and failure from a self-regulation perspective have suggested additional avenues for translational interventions. It is well known that individuals who are suffering from depression or anxiety often ruminate over past failures by engaging in counterfactual thinking ("What might I have done differently?"), which in turn worsens their symptoms (Nolen-Hoeksema, 2000). The question is, how can the damaging effects of such ruminative thinking be reduced? We explored the possibility of stimulating the use of a mismatched regulatory thought process (regulatory non-fit) as a technique to reduce strength of engagement in ruminative responses to failure. This intervention technique is intended to reduce individuals' confidence in their interpretation of their own failures to live up to a personal standard. This technique, based on the self-regulation model, might be a valuable tool for the therapeutic goal of interrupting the cycle of rumination that can exacerbate and prolong both mood and anxiety disorders.

Table I PANAS Positive and Negative Affect Scores Before versus After Microintervention to Alter Strength of Engagement in the Promotion versus Prevention Systems

\begin{tabular}{|c|c|c|c|c|}
\hline \multicolumn{5}{|l|}{ Pre-Intervention } \\
\hline PANAS-NA & 21.02 & 20.77 & 22.01 & 21.15 \\
\hline \multicolumn{5}{|c|}{ Post-Intervention } \\
\hline PANAS-PA & 19.58 & 23.95 & 19.01 & 23.22 \\
\hline
\end{tabular}


The general hypothesis tested in the next pilot study was that defining failure as not attaining a gain is more important to promotion-focused individuals and more salient in depressive states, whereas defining failure as not avoiding a loss is more important to prevention-focused individuals and more salient in anxious states. Based on this general hypothesis, we developed a novel intervention technique for individuals with dysphoric versus anxious symptoms. As noted, it is common for individuals who are suffering from depression or anxiety to ruminate over past failures by engaging in counterfactual thinking. The purpose of the study was to explore how the damaging effects of such counterfactual rumination might be reduced. The study used the principle of regulatory non-fit as a basis for specifically targeted interventions to reduce strength of engagement in ruminative responses to failure. We tested this principle by having participants engage in a form of counterfactual thinking that was a poor fit with their promotion or prevention orientation.

We hypothesized that participants who reported significant levels of dysphoric symptoms, which are related to chronic failure in the promotion system, were likely to produce counterfactual thinking about correcting a past error of omission via an additive counterfactual ("What did I fail to do?"). Such a counterfactual, however, would be consistent with the hypomotivated state resulting from chronic promotion failure. We predicted that inducing individuals with chronic promotion failure (and hence, dysphoric symptoms) to generate subtractive counterfactuals for past failures instead (a poor fit to the promotion system) would acutely lessen their dysphoria by momentarily engaging the prevention system and providing a "boost" of motivational activation. Similarly, participants who reported significant levels of anxious symptoms, which are related to chronic failure in the prevention system, were likely to produce counterfactual thinking about correcting a past error of commission via a subtractive counterfactual (e.g., "What did I do that was wrong?"). Such a counterfactual, however, would aggravate the hypervigilant state resulting from chronic prevention failure. We predicted that inducing individuals with chronic prevention failure (and hence, anxiety symptoms) to generate additive counterfactuals for past failures instead (a poor fit to the prevention system) would acutely lessen their anxiety by decreasing prevention engagement strength.

Participants responded to flyers advertising a study about personality and motivation. Eighty-nine respondents participated in the first part of the study, consisting of questionnaires that included measures of chronic dysphoric and anxious emotions and symptoms. Of those participants, 59 fit into one of three groups to be recruited for the second visit (dysphoric, anxious, nondistressed) and completed the second session a minimum of two weeks later. At that session, participants completed questionnaires and several writing prompts, the latter varying according to which of several possible conditions the participant was randomly assigned.

At the start of the second visit, participants completed a 24-item reduced version of the Multiple Affect Adjective
Check List. Participants were then asked to describe in writing either (1) a problem or hassle from the past week that made them feel down and depressed, or (2) a problem or hassle from the past week that made them feel anxious and nervous. All participants in the dysphoric subgroup, and half of the participants in the nondistressed subgroup, wrote about a problem that made them feel down and depressed; all participants in the anxious subgroup, and the other half of the participants in the nondistressed subgroup, wrote about a problem that made them feel anxious and nervous. Participants were then randomly assigned to write about either an additive counterfactual to the problem ("Now think about what action you could have taken, what you could have done that you did not do, that would have been more successful"), a subtractive counterfactual to the problem ("Now think about what you did to deal with the problem that was a mistake. What might you have done differently?"), or a no-writing condition in which participants simply waited. After writing about the assigned type of problem, participants completed an identical 24-item mood checklist. All participants then were given two opportunities for written rumination, with the first asking participants to recount their problem and add any additional information as desired, and the second asking how the problem had been or might be resolved. Finally, participants completed a third mood rating.

The main study hypotheses concerned the acute emotional impact of engaging distressed participants in counterfactual thinking that was a poor fit with their immediate negative motivational state - that is, the type of counterfactual did not fit with the type of goal they believed they were failing to attain (promotion vs. prevention) and the accompanying type of distress (dysphoric vs. anxious, respectively). Hierarchical linear analyses were conducted examining change in dysphoric and anxious mood from the start of the session through the priming and counterfactual writing condition and the additional ruminative writing stage.

We predicted that anxious individuals would experience a decrease in anxious mood after writing about an additive counterfactual, which is a non-fit to the motivational system (the prevention system) that is hypothesized to be dysfunctional for them. We likewise predicted that dysphoric individuals would experience a decrease in sad mood after writing about a subtractive counterfactual, which is a non-fit to the motivational system (the promotion system) that is dysfunctional for them. An omnibus hierarchical linear model was tested with Time (pre, post-counterfactual, post-additional writing) as a within-subject factor and Writing Condition (sad, anxious) and Counterfactual Condition (subtractive, additive, none) as between-subject factors. The linear trend was significant but no significant quadratic trend was found, so the analyses focused on linear patterns of change across the three measurement points. A significant Time $\times$ Counterfactual interaction was observed, $t(56)=2.21, p<.05$, which was moderated by a significant Time $\times$ Writing Condition $\times$ Counterfactual interaction, $t(56)=2.49, p<.05$. 


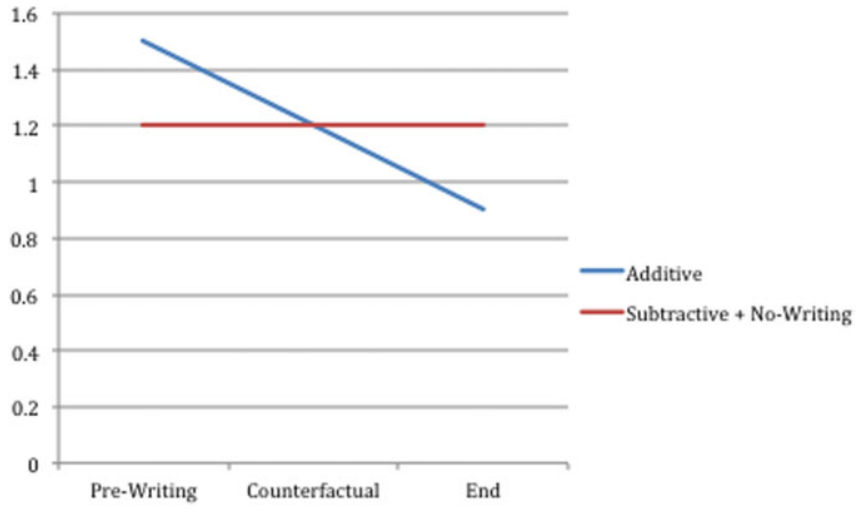

Figure IA Change in anxious mood by counterfactual condition.

The significant three-way interaction reflected two distinct patterns of change within the subsets of participants. For the additive counterfactual condition, we observed that the anxious participants showed a significant decrease in selfreported anxious mood across the three time points, $t(55)=2.19, p<.05$, whereas neither the dysphoric nor the control participants manifested such a decrease (both showed no statistically significant positive or negative change in anxious mood). In contrast, for the subtractive counterfactual condition, we observed that the dysphoric participants showed a significant decrease in self-reported sad mood across the three time points, $t(55)=2.33, p<.05$, whereas neither the anxious nor the control participants manifested such a decrease (both showed no statistically significant positive or negative change in dysphoric mood). The figures (combined across groups for ease of interpretation) show the estimated average slope of self-reported anxious mood change for the subtractive counterfactual compared with the additive counterfactual plus the no-writing condition (Figure 1A) as well as the estimated average slope of sad mood change for the additive counterfactual compared with the subtractive counterfactual plus the no-writing condition (Figure 1B).

Based on these two pilot studies, it appears that with guided instruction, individuals are able to interrupt and reduce selfregulation-related distress via techniques that target characteristics of the promotion and prevention systems. Part of the novelty of these two micro-interventions is that in one case, the therapist using an RFT-based technique would be seeking to help the individual increase engagement in adaptive selfregulation, while in the other the intent is to decrease engagement in maladaptive self-regulation. The strategies are direct translations of basic science findings in the self-regulation literature, and if subsequent research indicates that they are effective in clinically diagnosed individuals, then they could easily be added to existing therapies such as SST. Of course, it should be stated clearly that such micro-level interventions would be situated within the broader context of therapy, such that they complement and reinforce the work of the patient and therapist as they explore the dominant themes of the individual's life.

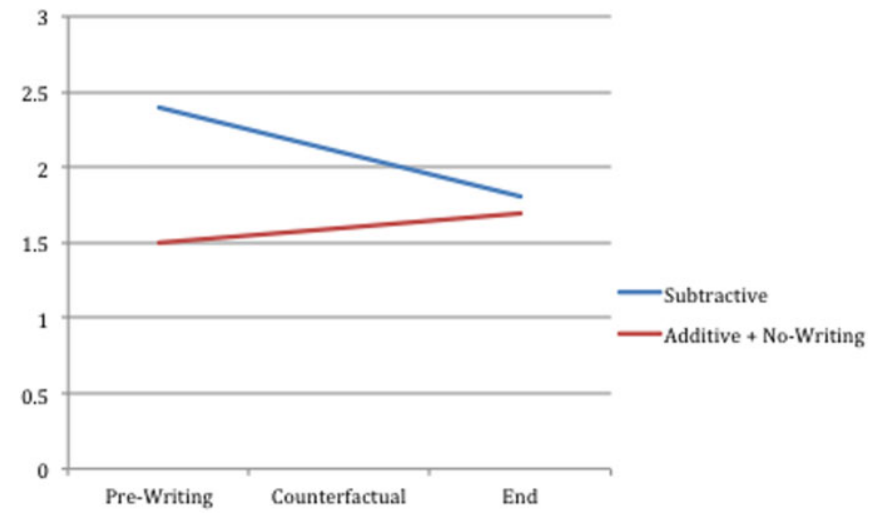

Figure IB Change in dysphoric mood by counterfactual condition.

Within self-system therapy, cognitive therapy, or interpersonal therapy, for example, there would likely be a continuing focus on examining the content and history of the individual's personal goals. We see these microinterventions as tools that can be brought to bear within the overall scope of a course of therapy, and particularly for problems in personal goal pursuit.

\section{Conclusion:Toward a Systematic Translational Paradigm in Psychotherapy Research}

From the very beginnings of research on psychotherapy, observers and investigators alike have called for the inclusion of cutting-edge theory and methodology to help elucidate the complex and interacting processes that account for the impact of therapy in people's lives (Kazdin, 2011). This perspective on psychotherapy research has existed in an uneasy truce with other traditions (Beutler, 2011; Lambert, 2011). In our view, this state of affairs results in part from confusion about the essence of psychotherapy. Is it a unique and powerful therapeutic relationship? A technology for behavior change? Should it be studied naturalistically or in the laboratory? Of course, such questions are simplistic and could never have either/or answers. Rather, it will continue to be important to pursue a better understanding of psychotherapy via multiple conceptual and methodological pathways, including the application and translation of basic science using rigorous laboratory-based methods where possible (Caspar, 2003).

Theories of individual differences, both psychological (e.g., Mischel, 2004) and neurobiological (e.g., Hariri, 2009), offer especially fertile conceptual ground for a systematic exploration of Paul's paradigmatic questions. For whom does psychotherapy A work, and does it work better than psychotherapy B for that individual? If so, why? What aspects of psychotherapy A are most salient or appropriate for a particular individual receiving it? If we can detect patterns of differential effectiveness within the available data, can we identify potential matches between the techniques or emphases of particular 
therapies and the needs (or strengths) of particular individuals? As we have argued throughout the paper, essential elements to gain traction regarding these questions include theory to provide an analytic framework and precise research designs to provide rigorous tests of key hypotheses.

Why is self-regulation a potentially valuable target for studying how psychotherapy works? We can at least offer opinion and conjecture here. First, as noted previously, selfregulation of goal pursuit is a critical locus for the impact of psychological and neurobiological processes on behavior and well-being. Individual characteristics as diverse as reward sensitivity, rumination, interpersonal style, need for achievement, and delay of gratification all are likely to influence how an individual perceives and responds to social interactions in terms of opportunities to "make something good happen" or "keep something bad from happening." Second, RFT is explicitly framed both as social psychology and as personality psychology; there are both context-driven and individually characteristic influences that lead people to construe events in terms of their own promotion and/or prevention goals. This dual personality/social nature of the theory, in turn, may offer multiple avenues for intervention within psychotherapy. Third, as the literature on self-regulation continues to expand (e.g., Hoyle, 2010), there will be more and more targets for intervention available to therapists who familiarize themselves with emerging theory and research. Perhaps it is fair to say that self-regulation also is a locus for the study of how social and personal processes interact with psychotherapy processparticularly if the treatment itself takes advantage of such knowledge.

The research we have summarized represents only a small step toward a systematic explication of how individual differences in self-regulation may be relevant to psychotherapy outcome and process. We are intrigued by several possibilities that we have only begun to explore. One is to build upon microintervention studies by integrating them with traditional randomized clinical trials - a "meso" level of analysis (with the "macro" level being analysis of data across multiple trials with multiple treatment modalities). Another is to investigate whether this self-regulation framework might also be useful as a prevention model, by identifying individuals vulnerable to self-regulatory dysfunction and intervening before disorders emerge. Yet another is to explore commonalities (and distinctions) between basic-science models of self-regulation and theories of universal relationship-based aspects of psychotherapy. At the least, our hope is that this paper offers an example of how such integrations might work, and illustrates how both basic science and the study of psychotherapy could benefit from true two-way translational research.

\section{References}

Austin, J. T., \& Vancouver, J. B. (1996). Goal constructs in psychology: Structure, process, and content. Psychological Bulletin, 120, 338-375.
Bandura, A. (2006). Toward a psychology of human agency. Perspectives on Psychological Science, 1, 164-180.

Belsky, J., \& Pluess, M. (2009). Beyond diathesis stress: Differential susceptibility to environmental influences. Psychological Bulletin, 135, 885-908.

Beutler, L. E. (2011). Prescriptive matching and systematic treatment selection. In J. C. Norcross, G. R. VandenBos, \& D. K. Freedheim (Eds.), History of psychotherapy (2nd ed., pp. 402-417). Washington, DC: American Psychological Association.

Beitman, B. D., Viamontes, G. I., Soth, A. M., \& Nittler, J. (2006). Toward a neural circuitry of engagement, self-awareness, and pattern search. Psychiatric Annals, 36, 272-280.

Brenner, H. D., Roder, V., \& Tschacher, W. (2006). Editorial: The significance of psychotherapy in the age of neuroscience. Schizophrenia Bulletin, 31, S10-S11.

Cacioppo, J., Berntson, G. G., Sheridan, J. F., \& McClintock, M. K. (2000). Multilevel integrative analyses of human behavior: Social neuroscience and the complementing nature of social and biological approaches. Psychological Bulletin, 126, 829-843.

Carrig, M. M., Kolden, G. G., \& Strauman, T. J. (2009). Using functional magnetic resonance imaging in psychotherapy research: A brief introduction to concepts, methods, and task selection. Psychotherapy Research, 19, 409-417.

Caspar, F. (1997). What goes on in a psychotherapist's mind? Psychotherapy Research, 7, 105-125.

Caspar, F. (2003). Psychotherapy research and neurobiology: Challenge, chance, or enrichment? Psychotherapy Research, 13, 1-23.

Castonguay, L. G. (1993). "Common factors" and "non-specific variables": Clarification of the two concepts and recommendations for research. Journal of Psychotherapy Integration, 3, 267-286.

Clark, D. M., Ehlers, A., Hackmann, A., McManus, F., Fennell, M., Grey, N., Waddington, L., \& Wild, J. (2006). Cognitive therapy versus exposure and applied relaxation in social phobia: A randomized controlled trial. Journal of Consulting and Clinical Psychology, 74, 568-578.

Connors, M. E. (2011). Attachment theory: A "secure base" for psychotherapy integration. Journal of Psychotherapy Integration, 21, 348-362.

Cuthbert, B. N., Schupp, H. T., Bradley, M. M., Birbaumer, N., \& Lang, P. J. (2000). Brain potentials in affective picture processing: Covariation with autonomic arousal and affective report. Biological Psychology, 52, 95-111.

Davidson, R. J., Jackson, D. C., \& Kalin, N. H. (2000). Emotion, plasticity, context, and regulation: Perspectives from affective neuroscience. Psychological Bulletin, 126, 890-909.

DeRubeis, R. J., Siegle, G. J., \& Hollon, S. D. (2008). Cognitive therapy versus medication for depression: Treatment outcomes and neural mechanisms. Nature Reviews Neuroscience, 9, 788-796.

Dozois, D. J. A., \& Beck, A. T. (2011). Cognitive therapy. In J. D. Herbert \& E. M. Forman (Eds.), Acceptance and mindfulness in cognitive behavior therapy: Understanding and applying the new therapies (pp. 26-56). Hoboken, NJ: John Wiley.

Elliot, A. J. (2008). The goal construct in psychology. In J. Y. Shah and W. L. Gardner (Eds.), Handbook of motivation science (pp. 235 250). New York, NY: Guilford Press. 
Etkin, A., Pittenger, C., Polan, H. J., \& Kandel, E.R. (2005). Toward a neurobiology of psychotherapy: Basic science and clinical applications. Journal of Neuropsychiatry and Clinical Neuroscience, 17, 145-158.

Forsyth, D. R., \& Strong, S. R. (1986). The scientific study of counseling and psychotherapy: A unificationist view. American Psychologist, 41, 113-119.

Goldapple, K., Segal, Z., Garson, C., Lau, M., Bieling, P., Kennedy, S., \& Mayberg, H. (2004). Modulation of cortical-limbic pathways in major depression: Treatment-specific effects of cognitive behavior therapy. Archives of General Psychiatry, 61, 34-41.

Grawe, H. (2007). Neuropsychotherapy: How the neurosciences inform effective psychotherapy. Mahwah, NJ: Lawrence Erlbaum.

Greenberg, L. (2008). Emotion and cognition in psychotherapy: The transforming power of affect. Canadian Psychology, 49, 49-59.

Hajcak, G., \& Nieuwenhuis, S. (2006). Reappraisal modulates the electrocortical response to unpleasant pictures. Cognitive, Affective, \& Behavioral Neuroscience, 6, 291-297.

Hariri, A. R. (2009). The neurobiology of individual differences in complex behavioral traits. Annual Review of Neuroscience, 32, 225-247.

Heatherton, T. F., \& Wagner, D. D. (2011). Cognitive neuroscience of self-regulation failure. Trends in Cognitive Sciences, 15, 132-139.

Higgins, E. T. (1987). Self-discrepancy: A theory relating self and affect. Psychological Review, 94, 319-340.

Higgins, E. T. (1998). Promotion and prevention: Regulatory focus as a motivational principle. Advances in Experimental Psychology, 46, 1-46.

Higgins, E. T. (2001). Promotion and prevention experiences: Relating emotions to nonemotional motivational states. In J. P. Forgas (Ed.), Handbook of affect and social cognition (pp. 186-211). Hillsdale, NJ: Lawrence Erlbaum.

Higgins, E. T., Marguc, J., \& Scholer, A. A. (2012). Working under adversity: How opposing versus coping affects value. Unpublished manuscript, Columbia University.

Higgins, E. T., \& Spiegel, S. (2004). Promotion and prevention strategies for self-regulation: A motivated cognition perspective. In R. F. Baumeister \& K. D. Vohs (Eds.), Handbook of self-regulation: Research, theory, and applications (pp. 171-187). New York, NY: Guilford Press.

Hollon, S. D., \& Ponniah, K. (2010). A review of empirically supported psychological therapies for mood disorders in adults. Depression and Anxiety, 27, 891-932.

Hoyle, R. H. (Ed.). (2010). Handbook of personality and selfregulation. Malden, MA: Blackwell.

Jacobson, N. S., Martell, C. R., \& Dimidjian, S. (2001). Behavioral activation treatment for depression: Returning to contextual roots. Clinical Psychology: Science and Practice, 8, 255-270.

Karoly, P. (1999). A goal systems-self-regulatory perspective on personality, psychopathology, and change. Review of General Psychology, 3, 264-291.

Kazdin, A. E. (2011). Evidence-based treatment research: Advances, limitations, and next steps. American Psychologist, 66, 685698.
Kim, S. H., \& Hamann, S. (2007). Neural correlates of positive and negative emotion regulation. Journal of Cognitive Neuroscience, 19, 776-798.

Lambert, M. J. (2011). Psychotherapy research and its achievements. In J. C. Norcross, G. R. VandenBos, \& D. K. Freedheim (Eds.), History of psychotherapy (2nd ed., pp. 299-332). Washington, DC: American Psychological Association.

Lewis, M. D., Lamm, C., Segalowitz, S. J., Stieben, J., \& Zelazo, P. D. (2006). Neurophysiological correlates of emotion regulation in children and adolescents. Journal of Cognitive Neuroscience, 18, 430-443.

Linden, D. E. J. (2006). How psychotherapy changes the brain: The contribution of functional neuroimaging. Molecular Psychiatry, 11, 528-538.

Lutz, W., Leach, C., Barkham, M., Lucock, M., Stiles, W.B., Evans, C., Noble, R. \& Iveson, S. (2005). Predicting rate and shape of change for individual clients receiving psychological therapy: Using growth curve modeling and nearest neighbor technologies. Journal of Consulting and Clinical Psychology, 73, 904913.

Lutz, W., Stulz, N., \& Köck, K. (2009). Patterns of early change and their relationship to outcome and follow-up among patients with major depressive disorders. Journal of Affective Disorders, 118, 60-68.

Miller, A. K., \& Markman, K. D. (2007). Depression, regulatory focus, and motivation. Personality and Individual Differences, 43, 427-436.

Miller, G. A. (2010). Mistreating psychology in the decades of the brain. Perspectives on Psychological Science, 5, 716-743.

Mischel, W. (2004). Toward an integrative science of the person. Annual Review of Psychology, 55, 1-22.

National Institute of Mental Health. (2000). Translating behavioral science into action: Report of the National Advisory Mental Health Council Behavioral Science Workgroup. Available at www.apps.nimh.nih.gov/ecb/archives/nimhtranslating.pdf

National Institutes of Health (NIH). (2007). Re-engineering the clinical research enterprise: Translational research. Available at http:// nihroadmap.nih.gov/clinicalresearch/overview-translational.asp

Nolen-Hoeksema, S. (2000). The role of rumination in depressive disorders and mixed anxiety/depressive symptoms. Journal of Abnormal Psychology, 109, 504-511.

Norcross, J. C., \& Wampold, B. E. (2011). What works for whom: Tailoring psychotherapy to the person. Journal of Clinical Psychology: In Session, 67, 127-132.

Ochsner, K. N., \& Gross, J. J. (2008). Cognitive emotion regulation: Insights from social cognitive and affective neuroscience. Currents Directions in Psychological Science, 17, 153-158.

Olofsson, J. K., Nordin, S., Sequeira, H., \& Polich, J. (2008). Affective picture processing: An integrative review of ERP findings. Biological Psychology, 77, 247-265.

Paul, G. (1967). Strategy of outcome research in psychotherapy. Journal of Consulting Psychology, 31, 109-118.

Peterson, C. K., Gable, P., \& Harmon-Jones, E. (2008). Asymmetrical frontal ERPs, emotion, and behavioral approach/inhibition sensitivity. Social Neuroscience, 3, 113-124. 
Phan, K. L., Fitzgerald, D. A., Nathan, P. J., Moore, G. J., Uhde, T. W., \& Tancer, M. E. (2005). Neural substrates for voluntary suppression of negative affect: A functional magnetic resonance imaging study. Biological Psychiatry, 57, 210-219.

Popper, K. R. (1979). Objective knowledge: An evolutionary approach. Oxford, UK: Oxford University Press.

Roffman, J. L., Marci, C. D., Glick, D. M., Dougherty, D. D., \& Rauch, S. L. (2005). Neuroimaging and the functional neuroanatomy of psychotherapy. Psychological Medicine, 35, 13851398.

Siegle, G. J., Thompson, W., Carter, C. S., Steinhauer, S. R., \& Thase, M. E. (2007). Increased amygdala and decreased dorsolateral prefrontal BOLD responses in unipolar depression: Related and independent features. Biological Psychiatry, 61, 198-209.

Strauman, T. J. (2002). Self-regulation and depression. Self and Identity, 1, 151-157.

Strauman, T. J., Klenk, M. M., and Eddington, K. M. (in press). Self-regulation as a mediator of change in psychotherapy. In D. Hermans, B. Rimé, \& B. Mesquita (Eds.), Changing emotions. London, UK: Psychology Press.

Strauman, T. J., Kolden, G., Davis, N., Stromquist, V., Kwapil, L., \& Heerey, E. (2001). The effects of treatments for depression on failure in self-regulation. Cognitive Therapy and Research, 25, 693-712.

Strauman, T. J., Vieth, A. Z., Merrill, K. A., Woods, T., Kolden, G. G., Woods, ... Kwapil, L. (2006). Self-system therapy as an intervention for self-regulatory dysfunction in depression: A random- ized comparison with cognitive therapy. Journal of Consulting and Clinical Psychology, 74, 367-376.

Sung, N. S., Crowley, W., Genel, M., Salber, P., Sandy, L., Sherwood, L., . . Rimoin, D. (2003). Central challenges facing the national clinical research enterprise. Journal of the American Medical Association, 289, 1278-1287.

Tangney, J. P., Niedenthal, P. M., Covert, M. V., \& Barlow, D. H. (1998). Are shame and guilt related to distinct self-discrepancies? A test of Higgins's (1987) hypotheses. Journal of Personality and Social Psychology, 75, 256-268.

Taylor, S. F., \& Liberzon, I. (2007). Neural correlates of emotion regulation in psychopathology. Trends in Cognitive Sciences, 11, 413-418.

Vieth, A., Strauman, T. J., Kolden, G., Woods, T., \& Klein, M. H. (2003). Self-system therapy: A theory-based psychotherapy for depression. Clinical Psychology: Science and Practice, 10, 245268.

Woolf, S. H. (2008). The meaning of translational research and why it matters. Journal of the American Medical Association, 299, 211213.

Zaunmüller, L., Lutz, W., \& Strauman, T.J. (under review). Affective impact and electrocortical correlates of a cognitive microintervention: An ERP study. Cognitive Therapy and Research.

Zinbarg, R. E., \& Griffith, J. W. (2008). Behavior therapy. In J. L. Lebow (Ed.), Twenty-first century psychotherapies: Contemporary approaches to theory and practice. Hoboken, NJ: John Wiley. 